Ines-Jacqueline Werkner - Martina Fischer (Hrsg.)

\title{
Europäische
}

Friedensordnungen

und Sicherheits-

architekturen

Politisch-ethische Herausforderungen Band 3

是 SpringerVS 
Hrsg.

Ines-Jacqueline Werkner

Martina Fischer

Heidelberg, Deutschland

Berlin, Deutschland

\section{Gerechter Frieden}

ISBN 978-3-658-23919-0

https://doi.org/10.1007/978-3-658-23920-6

ISBN 978-3-658-23920-6 (eBook)

Die Deutsche Nationalbibliothek verzeichnet diese Publikation in der Deutschen Nationalbibliografie; detaillierte bibliografische Daten sind im Internet über http://dnb.d-nb.de abrufbar.

Springer VS

(c) Springer Fachmedien Wiesbaden GmbH, ein Teil von Springer Nature 2019

Das Werk einschließlich aller seiner Teile ist urheberrechtlich geschützt. Jede Verwertung, die nicht ausdrücklich vom Urheberrechtsgesetz zugelassen ist, bedarf der vorherigen Zustimmung des Verlags. Das gilt insbesondere für Vervielfältigungen, Bearbeitungen, Übersetzungen, Mikroverfilmungen und die Einspeicherung und Verarbeitung in elektronischen Systemen.

Die Wiedergabe von Gebrauchsnamen, Handelsnamen, Warenbezeichnungen usw. in diesem Werk berechtigt auch ohne besondere Kennzeichnung nicht zu der Annahme, dass solche Namen im Sinne der Warenzeichen- und MarkenschutzGesetzgebung als frei zu betrachten wären und daher von jedermann benutzt werden dürften.

Der Verlag, die Autoren und die Herausgeber gehen davon aus, dass die Angaben und Informationen in diesem Werk zum Zeitpunkt der Veröffentlichung vollständig und korrekt sind. Weder der Verlag, noch die Autoren oder die Herausgeber übernehmen, ausdrücklich oder implizit, Gewähr für den Inhalt des Werkes, etwaige Fehler oder Äußerungen. Der Verlag bleibt im Hinblick auf geografische Zuordnungen und Gebietsbezeichnungen in veröffentlichten Karten und Institutionsadressen neutral.

Verantwortlich im Verlag: Jan Treibel

Springer VS ist ein Imprint der eingetragenen Gesellschaft Springer Fachmedien Wiesbaden $\mathrm{GmbH}$ und ist ein Teil von Springer Nature

Die Anschrift der Gesellschaft ist: Abraham-Lincoln-Str. 46, 65189 Wiesbaden, Germany 


\title{
Die Rolle des Vertrauens für eine Sicherheitspolitik der Kooperation
}

\author{
Pascal Delhom
}

\section{$1 \quad$ Einleitung}

Der Schutz vor Gewalt und Verletzungen ist ohne Zweifel eine der wichtigsten Aufgaben jeder Friedens- und Sicherheitspolitik. Denn kein Frieden kann von Dauer sein, in dem ein solcher Schutz nicht gewährleistet wird und in dem sich Menschen vor anderen Menschen fürchten müssen. Dies gilt umso mehr für eine Auffassung des gerechten Friedens, die nicht nur vor Gewalt, sondern auch vor Not zu schützen beansprucht. Wie allerdings dieser Schutz gewährt wird, hängt weitgehend von der Auffassung von Sicherheit ab, die ihn realisieren soll. Ich werde mich im Folgenden auf eine Sicherheit durch Kooperation konzentrieren, die mir als einzige im Rahmen einer Politik des Friedens angemessen zu sein scheint, die weder gegen noch ohne die anderen, sondern nur mit ihnen geführt werden kann. Eine der Bedingungen einer entsprechenden Sicherheitspolitik ist das Vertrauen, das allerdings selber nicht bedingungslos geschenkt und nicht ohne Voraussetzungen erworben werden kann. Um die verschiedenen Dimensionen 
einer Praxis des Vertrauens im Dienst der Sicherheit wird es im folgenden Beitrag gehen.

\section{Sicherheitspolitik - zwei Perspektiven}

Seit geraumer Zeit orientiert sich der Begriff der Sicherheitspolitik - im theoretischen wie im politischen Diskurs - nach einem bestimmten, gefahrenzentrierten Verständnis von Sicherheit. Erläuterungen zur Sicherheitspolitik bemühen meistens das semantische Feld der Gefahrenabwehr und rechtfertigen entsprechende Maßnahmen, auch wenn diese auf Kosten der eigenen Freiheit erfolgen. Dennoch möchte ich die folgenden Überlegungen mit einer sehr schematischen, aber heuristisch wichtigen Unterscheidung zwischen zwei Grundarten der Sicherheitspolitik einleiten. Dass keine Politik und keine Sicherheitsinstitution ausschließlich der einen Art entsprechen, vermag nicht darüber hinwegzutäuschen, dass es sich um entgegengesetzte Verständnisse handelt.

Die erste Art ist eben gefahrenzentriert. Sie besteht erstens in der Definition möglicher und realer Gefahren für eine gesellschaftliche Ordnung, zweitens in der Verortung der Quellen dieser Gefahren innerhalb und außerhalb dieser Ordnung und drittens in der Bekämpfung dieser Gefahren durch Ein- und Aussperrung, durch Abschreckung oder durch Zerstörung dieser Quellen. Auf allen Ebenen ist eine solche Sicherheitspolitik mit Gewalt behaftet, sei es in der Form einer einbrechenden, verletzenden und tötenden, einer ausschließenden oder einer angedrohten Gewalt, sei es eine Gewalt der Waffen, der Überwachung oder sogar der drohenden beziehungsweise als "Gefährder" definierenden Sprache. Auf allen Ebenen ist diese Sicherheitspolitik auch freiheitsberaubend, und zwar nicht nur für diejenigen, die beseitigt, ein- und ausgesperrt, überwacht, bedroht oder als potenzielle Gefährder kategorisiert 
werden, sondern auch für diejenigen, die durch sie geschützt werden sollen, die aber in der ständigen Angst vor diesen Gefahren leben müssen, denn ohne diese Angst würde die gefahrenzentrierte Sicherheitspolitik jede Legitimierung ihrer Gewaltsamkeit und der mit ihr einhergehenden Einschränkung der Freiheit verlieren. Eine gefahrenzentrierte Sicherheitspolitik muss also das Bewusstsein der drohenden Gefahr aufrechterhalten, als Garantie ihrer Notwendigkeit und ihrer Legitimität. Allerdings schränkt dieses Bewusstsein selbst die Handlungsfreiheit ein: Freies Handeln setzt nämlich ein Gefühl der Sicherheit voraus und wird durch Angst systematisch untergraben (vgl. Montesquieu 1951 [1748], Buch XII).

Demgegenüber steht eine Auffassung der Sicherheit durch Kooperation, die nicht konstitutiv gegen andere gerichtet, sondern im Gegenteil als eine gemeinsame Aufgabe aufgefasst wird, die jede Seite nur mit der anderen erfüllen kann. Eine solche Sicherheitspolitik fasst entsprechend die anderen nicht primär als (potenzielle) Gefahren auf. Sie besteht vielmehr darin, die Bedingungen zu verwirklichen, unter denen sie jeweils für uns keine Gefahr darstellen beziehungsweise nicht zu einer Gefahr werden. Dies setzt erstens voraus, dass die anderen als unverzichtbare Akteure einer gemeinsamen Sicherheitspolitik anerkannt werden. Es fordert zweitens, dass Praktiken und Strategien des gemeinsamen Handelns gemeinsam entwickelt werden, die eine verlässliche Grundlage für die Wahrnehmung der gemeinsamen Aufgabe der Sicherheit bilden. Eine solche Sicherheitspolitik vermeidet den Rekurs auf sei es nur drohende - Gewalt gegen die Kooperationspartner, der die Bedingungen des gemeinsamen Handelns nur gefährden kann. Und sie ist insofern freiheitsfördernd, als jede Partei die Freiheit der anderen anerkennen muss und diese sogar zur Erfüllung der gemeinsamen Aufgabe einbezieht und einsetzt.

Eine auf Kooperation basierende Sicherheitspolitik ist notwendig eine langfristig angelegte Politik. Sie scheint in den meisten 
Fällen unangebracht, wenn es darum geht, unmittelbar drohende Gefahren abzuwehren. Mitten in einem bewaffneten Konflikt oder in Anbetracht terroristischer Bedrohung kann sie ja sogar als blauäugig und gefährlich erscheinen. Allerdings erfordert sie, dass auch im Fall einer unmittelbaren Gefahrenabwehr keine Mittel eingesetzt werden, die eine zukünftige Kooperation unmöglich machen. Ähnlich hatte bereits Immanuel Kant (1968 [1795], S. 200) in seinem sechsten Präliminarartikel zum ewigen Frieden gefordert

\begin{abstract}
„Es soll sich kein Staat im Kriege mit einem anderen solche Feindseligkeiten erlauben, welche das wechselseitige Zutrauen im künftigen Frieden unmöglich machen müssen [...]. Denn irgend ein Vertrauen auf die Denkungsart des Feindes muß mitten im Kriege noch übrig bleiben, weil sonst auch kein Friede abgeschlossen werden könnte, und die Feindseligkeit in einen Ausrottungskrieg ausschlagen würde."
\end{abstract}

Aber auch in manchen Fällen der unmittelbaren Bedrohung kann eine Sicherheitspolitik der Kooperation, wenn sie konsequent durchgeführt wird, wirksam und wünschenswert sein. So beschreibt der General Jacques Pâris de Bollardière (1972, S.77ff.), wie er als junger General mitten im Algerienkrieg von 1956 bis 1958 eine systematische Politik der Kooperation zwischen allen Bevölkerungsgruppen in dem algerischen Teilgebiet durchführte, das unter seinem Kommando stand. Neben dem absoluten Verbot der Folter und der willkürlichen Gewaltanwendung, die sonst von der französischen Armee in Algerien eingesetzt wurden, bemühte er sich um Praktiken des Respekts der anderen und um das Gewinnen ihres Vertrauens. Bald konnte er auch den Aufbau von Infrastruktur und Handelsbeziehungen vorantreiben, die vom Krieg unterbrochen worden waren. Nach einigen Monaten waren die terroristischen Angriffe in diesem Gebiet erheblich zurückgegangen. 1958 wurde er abgesetzt, als er sich weigerte, die 
Methoden des Generals Massu - unmittelbare Effizienz, Schutz von Menschenleben um jeden Preis, auch unter Einsatz von Folter - zu billigen und anzuwenden. Seine zweijährigen Anstrengungen wurden in nur wenigen Wochen zerstört und der Terrorismus kehrte wieder zurück.

Doch es liegt nahe, dass eine solche Sicherheitspolitik, auch wenn sie als wünschenswert erscheinen mag, nicht ohne Bedingungen möglich ist. Eine dieser Bedingungen, auf die ich mich im Folgenden konzentrieren möchte, ist der bereits angesprochene Aufbau des Vertrauens. Denn Kooperation ist als solche nur als gegenseitiges Einbeziehen und Einsetzen des freien Handelns der jeweils anderen, der weder beherrscht noch systematisch überwacht wird, möglich. Und Vertrauen kann als eine privilegierte Form des Umgangs mit ebendieser Freiheit der anderen, das heißt mit ihrem freiwilligen Handeln und Verhalten angesehen werden.

\section{Vertrauen 1}

Auch wenn immer wieder in politischen und vor allem in wirtschaftlichen Diskursen von den Vorteilen eines gegenseitigen Vertrauens die Rede ist, ist Vertrauen grundsätzlich eine asymmetrische Beziehung. Denn das jeweils eigene Vertrauen, das wir im anderen haben oder das wir ihm schenken, hängt nicht vom Vertrauen des anderen in mir ab. Und das Wagnis des Vertrauens wird nicht dadurch geringer, dass der andere dasselbe Wagnis auch eingeht. Gegenseitiges Vertrauen bildet also keine Symmetrie der Beziehung, sondern eine doppelte Asymmetrie. Auf der Seite derjenigen, denen Vertrauen geschenkt wird, eröffnet dieses Vertrauen

1 Zur Unmöglichkeit einer restlosen Begründung des Vertrauens vgl. auch Delhom (2018). 
einen Spielraum des Handelns, innerhalb dessen nicht jede Geste geprüft, jede Entscheidung kontrolliert oder sogar vorgegeben wird. Vertrauen setzt also jemanden in die Verantwortung für eine bestimmte Aufgabe ein und ermöglicht ihm oder ihr die freie Ausübung dieser Verantwortung. Auf der Seite des Vertrauenden besteht wiederum das Vertrauen darin, dass es die Komplexität des eigenen Handelns in Anbetracht des freien Handelns der anderen reflektiert und reduziert (vgl. Luhmann 2000): Es erlaubt uns, Entscheidungen zu treffen und entsprechend $\mathrm{zu}$ handeln, ohne uns in Bezug auf alle möglichen Handlungen der anderen abgesichert zu haben.

Für die Vertrauenden bezieht sich das Vertrauen erstens auf die Fähigkeit anderer, bestimmte Handlungen durchzuführen oder bestimmte Aufgaben zu übernehmen. In diesem Sinne trauen wir ihnen zu, dass sie die Aufgaben, die wir ihnen anvertrauen, auch erfüllen können. Sonst würde unser Vertrauen sie überfordern und es wäre zum Scheitern verurteilt. Aber das Vertrauen bedeutet mehr als dieses Zutrauen in Bezug auf eine bestimmte Fähigkeit. Wir trauen nämlich durchaus manchen Leuten $\mathrm{zu}$, dass sie uns anlügen oder betrügen. Wir würden ihnen aber natürlich nicht vertrauen. Im Vertrauen erwarten wir darüber hinaus, dass sie in unserem Sinne handeln, obwohl wir nicht von vornherein alles festgelegt haben, was sie tun sollen, und wir nicht alle Elemente ihres Handelns kontrollieren (können). Das Vertrauen öffnet dem anderen einen Spielraum des Handelns, von dem wir nun annehmen, dass er in unserem Sinne benutzt wird.

Dieser Spielraum ist meistens nicht beliebig offen. Es stimmt zwar, dass wir bestimmten Menschen - manchen Mitgliedern unserer Familie oder Freunden - uneingeschränkt vertrauen. Meistens vertrauen wir aber Menschen nicht uneingeschränkt, sondern in Bezug auf bestimmte Aufgaben, die wir ihnen anvertrauen. Allerdings bedeutet diese Einschränkung in Bezug auf das Objekt des 
Vertrauens nicht, dass das Vertrauen selbst geringer sein soll. Denn die Aufgaben, um die es geht, sind nicht selten solche, die uns in unserem Leben am wichtigsten zu sein scheinen: Wir vertrauen Lehrer und Lehrerinnen die Erziehung unserer Kinder an, Ärzte und Ärztinnen unsere Gesundheit und sogar unser Leben, Politiker und Politikerinnen die Organisation unseres gemeinsamen Lebens usw. Eine dieser Aufgaben besteht eben auch darin, dass andere Menschen mit uns für unsere Sicherheit sorgen. Und die Menschen, denen wir diese Aufgabe anvertrauen, sind nicht nur Freunde und Verwandte.

Auf einer Mikroebene können diese Menschen Nachbarn sein, von denen wir uns nicht abschirmen, sondern mit denen wir - auch im Fall von Konflikten und als Ansatz zu deren Lösung - gute Nachbarschaftsbeziehungen zu pflegen versuchen. Aufgrund dieser Beziehungen tendieren wir dazu, ihre Präsenz nicht als eine Bedrohung zu empfinden, sondern im Gegenteil mit der Erwartung zu verbinden, dass sie für uns da sein werden, wenn wir sie brauchen. Dies trägt nicht nur objektiv zur Sicherheit einer Nachbarschaft bei, sondern es verleiht den Beteiligten ein Gefühl der Sicherheit, das keine Überwachungskamera und kein dreifaches Schloss zu verleihen vermag. Denn diese verweisen immer auf die Gefahren, vor denen sie schützen, wohingegen jene auf die Ressourcen verweisen, mit denen wir im Fall eines Problems rechnen können. Die wahrnehmbaren Lebenszeichen der Nachbarn werden dann allgemein und in Bezug auf unsere Sicherheit als beruhigend empfunden, auch wenn sie manchmal störend sein mögen.

Aber auch auf anderen Ebenen des Handelns und des Zusammenlebens, bis hin zur Makroebene der internationalen Politik, können wichtige Aufgaben der Sicherheit im Sinne einer Kooperation aufgefasst und angegangen werden. Ein solcher Ansatz bedeutet, dass die involvierten Parteien nicht primär die eigene Sicherheit durch Abschirmung, Überwachung und Abschreckung (auch im 
Rahmen von Bündnissen) zu garantieren versuchen, sondern die Aufgaben einer gemeinsamen Sicherheit ${ }^{2}$ gerade denjenigen mit anvertrauen, vor denen sie sich ansonsten fürchten würden und $\mathrm{zu}$ Recht fürchten müssten. Dies erfolgte zum Beispiel während des Kalten Krieges durch die erste Konferenz zur Sicherheit und Zusammenarbeit in Europa (KSZE, 1973-1975) und ihre wiederholten Folgekonferenzen, in denen nicht nur wichtige Entscheidungen über Abrüstung, Anerkennung der Souveränität der beteiligten Staaten, Verzicht auf Gewaltanwendung oder gegenseitige (Einladungen zur) Beobachtung von militärischen Aktivitäten getroffen wurden, sondern diese ausdrücklich als Vertrauens- und Sicherheitsbildende Maßnahmen (so der Titel der Stockholmer Konferenz von 1984-1986) verstanden wurden, die, zusammen mit Programmen der wirtschaftlichen und humanitären Zusammenarbeit maßgeblich zum Ende des Ost-West-Konflikts beigetragen haben (vgl. Czempiel 2012; Senghaas 1992).

Das Vertrauen, das im Rahmen solcher Sicherheitskooperationen benötigt und gebildet wird, ist nicht die Grundhaltung des Vertrauens, das wir in bestimmten Personen - etwa in Vertrauenspersonen seit der frühen Kindheit - haben. Es ist ein Vertrauen, das einerseits den anderen unter bestimmten Bedingungen geschenkt wird und das dadurch zur Etablierung und Festigung einer verbindlichen Beziehung beiträgt, in der Kooperation gewagt werden kann; es ist andererseits ein Vertrauen, das bei anderen durch bestimmte Handlungs- und Verhaltensweisen hervorgerufen wird, so dass auch er oder sie die Kooperation als Handlungsform bevorzugt. Ich wende mich nun diesen zwei Seiten der Vertrauensbildung zu.

2 Zum Konzept der gemeinsamen Sicherheit vgl. auch den Beitrag von Ines-Jacqueline Werkner in diesem Band. 


\section{Vertrauen schenken}

Das Schenken von Vertrauen birgt immer die Gefahr, dass es enttäuscht wird, dass die Schenkenden, die sich durch ihr Vertrauen verletzbar machen, eben dadurch verletzt werden und dass somit auch die Bedingungen ihres zukünftigen Vertrauens geschwächt werden. Vertrauen ist immer ein Wagnis, und zwar auch deswegen, weil es nie endgültig begründet werden kann. ${ }^{3}$ Deshalb ist es wichtig, einerseits Bedingungen zu berücksichtigen, unter denen Vertrauen geschenkt werden kann, andererseits gute Gründe des Misstrauens auch ernst zu nehmen. Das "gesunde" Misstrauen ist hier nicht das Gegenteil des Vertrauens, sondern seine notwendige Ergänzung. Es verbietet nicht das Vertrauen, sondern stärkt es umgekehrt mit der Forderung, so viel wie nötig zu misstrauen, um so weit wie möglich vertrauen zu können. Wer blind vertraut, gefährdet nicht nur sich selbst, sondern auch die Möglichkeit des Vertrauens.

Unter den Bedingungen des Vertrauens, die also berücksichtigt und gegebenenfalls gebildet werden müssen, zählt erstens die Annahme der Vertrauenswürdigkeit des jeweils anderen. Diese Annahme entsteht aus sehr unterschiedlichen Quellen: aus der Erfahrung vergangener Handlungen, aus dem Ruf einer Person, aus ihrem Aussehen (wir tendieren nämlich statistisch dazu, unseresgleichen mehr zu vertrauen als Fremden!), aber auch aus ihrer ausdrücklichen Selbstverpflichtung, ihrer vergangenen oder aktuellen Übernahme von Verantwortung oder einfach aus einer günstigen Situation (vgl. Sztompka 1999; Delhom 2014). Auch die Vertrautheit eines begrenzten sozialen Zusammenhangs („Wir

3 Wenn Vertrauen völlig begründet wäre, würde es sich selbst als Vertrauen aufheben. Wir bräuchten kein Vertrauen mehr. Was allerdings begründet werden muss und kann, ist das Misstrauen. 
werden uns sicher wiedersehen.“) kann Vertrauensbeziehungen begünstigen.

Eine zweite Absicherung des geschenkten Vertrauens liegt in einer eigentümlichen Normativität, die dem Vertrauen selbst innewohnt. Indem wir es schenken, machen wir uns nämlich vom anderen abhängig und durch ihn verwundbar, rufen aber gerade dadurch bei ihm die Verantwortung hervor, dass er unser Vertrauen nicht enttäuscht (vgl. Luhmann 2000, S. 55). In diesem Sinne kann behauptet werden, dass zumindest zu einem bestimmten Grad Vertrauen verpflichtet. Allerdings gilt dies nur in Gesellschaften, in denen das Ausnutzen des Vertrauens selbst verpönt und nicht als eine verdiente Bestrafung von Dummheit angesehen wird.

Deswegen zählt als eine dritte Bedingung des Vertrauens das, was eine „Kultur“ (Sztompka 1999), ein „Klima“ (Baier 1994) oder ein „Ethos“ (Röttgers 2004) des Vertrauens genannt werden kann. Es handelt sich um einen sozialen Habitus des Vertrauens, der eine günstige und begünstigende Grundlage des geschenkten Vertrauens bildet. In einer Gesellschaft, in der Vertrauen breit praktiziert und einen hohen Stellenwert hat, ist die verbindliche Kraft des geschenkten Vertrauens höher und entsprechend die Gefahr, betrogen zu werden, geringer als in einer solchen, in der das Vertrauen für dumm und der Vertrauende für blauäugig gehalten werden (vgl. Fukuyama 1995, der zwischen "high-trust" and "low-trust societies" unterscheidet). Umgekehrt tragen wiederum Erfahrungen des enttäuschten Vertrauens zur Zerstörung des Vertrauensklimas bei, wohingegen Erfahrungen des wiederholt eingesetzten und nicht enttäuschten Vertrauens zur Steigerung sowohl der Vertrauenswürdigkeit des anderen als auch des allgemeinen Vertrauensklimas beitragen. Zwischen dem Schenken des Vertrauens und dem ihm begünstigenden Klima besteht also ein Verhältnis der gegenseitigen Bestimmung. 
Letztlich kann viertens das Schenken des Vertrauens durch die Einbeziehung eines Dritten begünstigt und stabilisiert werden. ${ }^{4}$ Dieser oder diese Dritte kann sehr unterschiedlich auftreten: Sie kann etwa Zeugin eines Versprechens oder eines Vertrags sein, die Rolle der Vermittlerin bei einer Einigung spielen oder eine Bürgschaft bei einer finanziellen Verpflichtung übernehmen. Sie kann als einzelne Person, als Gruppe oder als Vertreterin einer Institution eintreten. Doch ihre Wirksamkeit besteht immer darin, dass sie der Zweierbeziehung der Vertrauenden und der Empfängerin des Vertrauens eine externe Perspektive hinzufügt, die diese strukturell (durch die Perspektivenverschiebung) und zeitlich (durch die Entstehung eines externen Gedächtnisträgers) stabilisiert. Beide Beteiligten werden sich auch in Zukunft auf diese Instanz berufen können. Zu beachten ist allerdings hierbei, dass diese Einbeziehung eines Dritten das Vertrauen nicht aufhebt, sondern verschiebt und ergänzt: Sie ermöglicht die Entwicklung einer Beziehung des Vertrauens, weil beide Seiten dem Dritten, etwa der Zeugin, vertrauen, die das Versprechen bezeugen kann. Wäre die Beziehung zum Dritten eine solche der Unterwerfung oder der Gewalt, würde dies auch das Vertrauen zum anderen zerstören beziehungsweise überflüssig werden lassen.

Diese Bedingungen des Vertrauens bilden - bereits einzeln, aber noch mehr durch ihre Zusammenwirkung - eine günstige Grundlage für ein Schenken des Vertrauens, das Sicherheit gewährt und nicht umgekehrt die Gefahr der eigenen Verletzung erhöht. Sie sind allerdings keine Garantie der Verlässlichkeit des anderen. Sie heben also nicht die grundsätzliche Asymmetrie des Vertrauensverhältnisses auf, das auch unter günstigen Bedingungen ein Wagnis bleibt. Deswegen ist es im Rahmen einer kooperativen

4 Über die vielfache Rolle des Dritten in sozialen Beziehungen vgl. Bedorf (2003). 
Sicherheitspolitik wichtig, dass die Beteiligten nicht nur unter Wahrung eines gesunden Misstrauens auf das Wagnis des Vertrauens eingehen, sondern dass sie selber überzeugende Zeichen der eigenen Vertrauenswürdigkeit senden.

\section{$5 \quad$ Vertrauen hervorrufen}

Besonders in Zeiten von Konflikten und Misstrauen können die Aufforderungen der einen Seite, die andere solle ihr vertrauen, eher eine abschreckende Wirkung haben. Der Ruf „vertraue mir“ wird geradezu als eine Bitte aufgefasst, sich wehrlos betrügen zu lassen. Auch Versprechen, die sich verbindlich geben, können allzu leicht als leere Worte verstanden werden, die nur diejenigen binden, die sie glauben.

In seiner Analyse des Endes des Kalten Krieges betont entsprechend Andrew H. Kydd (2007, S. 214ff.) die Wichtigkeit von „kostspieligen Zeichen“, durch die eine der Parteien die Verlässlichkeit ihrer Haltung gegenüber der anderen glaubwürdig zu bezeugen hat. Je geringer das Vertrauen des anderen ist, desto kostspieliger müssen die Signale sein, um zu überzeugen; je schwächer auch die Partei ist, die das Signal sendet, gegenüber der anderen Partei, gilt genau das Gleiche.

Am Ende des Kalten Krieges war nun das Vertrauen des Westens und besonders der Vereinigten Staaten in die Sowjetunion sehr gering und - vor allem aus ökonomischen Gründen - die Position der letzteren eher eine der Schwäche. In den Augen sowohl der US-amerikanischen Regierung als auch der Bevölkerung des Landes und Europas sprach also wenig dafür, die Signale des neuen Generalsekretärs des Zentralkomitees der Kommunistischen Partei der Sowjetunion Michail Gorbatschows in Richtung einer Entspannung, einer Liberalisierung des Landes und einer Abrüstung 
als glaubwürdig einzuschätzen. Es bedurfte vieler, zum Teil sehr kostspieliger Gesten seitens der UdSSR, um den Westen, der von einer ungebrochenen Expansionsstrategie ausging, zu beruhigen. Das 1985 beschlossene Moratorium über Atomtests und der Verzicht auf die Stationierung von SS-20 Mittelstreckenraketen schlugen in dieser Hinsicht fehl, weil sie als eine Geste angesehen wurden, die den Sowjets nicht wirklich wehtat (Kydd 2007, S. 225). Auch die Ergebnisse eines Treffens zwischen Ronald Reagan und Michail Gorbatschow im selben Jahr in Genf über eine Reduktion der strategischen Atomwaffen hatten nicht die erwünschte Wirkung. Erst die Annahme durch Gorbatschow, im Frühjahr 1987, eines bedingungslosen Rückzugs aller Mittelstreckenraketen von Europa - für den er noch bei einem Treffen in Reykjavik 1986 Bedingungen gestellt hatte - und die Bereitschaft der Sowjetunion, viel mehr Raketen zu zerstören als die Vereinigten Staaten, brachte einen ersten Durchbruch in Sache Vertrauen. 1988 trugen vier Ereignisse zur Überzeugung des Westens von den tiefen Veränderungen in der Sowjetunion bei (vgl. Kydd 2007, S.230ff.): Der Anfang des Rückzugs von Afghanistan, der Besuch Reagans in Moskau, der sich selber von den Veränderungen im Land überzeugen konnte, die neunzehnte Konferenz der Partei, in der Gorbatschow grundlegende politische, rechtliche und ökonomische Reformen ankündigte, und die Rede Gorbatschows vor der Generalversammlung der Vereinten Nationen, in der er einen substanziellen und unilateralen Truppenabbau in Osteuropa ankündigte.

Wichtig ist in dieser Entwicklung, dass den Ankündigungen von Gorbatschow auch Taten folgten, die für die andere Seite sichtbar und kontrollierbar waren, und dass diese Taten nicht mehr, wie am Anfang des Prozesses, als Teil einer Strategie der verdeckten sowjetischen Expansion gedeutet werden konnten. Kydd konstatierte, dass sich auch das persönliche Verhältnis zwischen Reagan und Gorbatschow in der Zeit gründlich änderte und ihr anfängliches 
tiefes Misstrauen allmählich dem Vertrauen wich. 1989, als George Bush Präsident der Vereinigten Staaten wurde, war der Prozess der Vertrauensbildung so weit vorangeschritten, dass sowohl in den westlichen Regierungen als auch in ihren Bevölkerungen das Vertrauen gegenüber dem Misstrauen bei weitem überwog. Ein „bedeutender Hinweis auf das neue Niveau des Vertrauens [war, dass] die Bush-Regierung auf ein Abkommen über Chemiewaffen ohne die gewöhnliche Betonung der Überprüfung drängte" (Kydd 2007, S. 234, Übers. d. Verf.). Umgekehrt fing auch die NATO an, Zeichen der Entspannung und der Beruhigung in die Richtung der Sowjetunion zu senden. Die Bedingungen einer grundsätzlichen Veränderung der Sicherheitspolitik zwischen den beiden Blöcken, die das Ende des Kalten Krieges einleiteten, waren realisiert. ${ }^{5}$

Der Hintergrund der Analyse von Kydd ist die Theorie der rationalen Wahl (rational choice), verbunden mit einer Version der Spieltheorie, die eine mögliche Wiederholung von Wahlentscheidungen einbezieht. Was er dabei entsprechend nicht berücksichtigt, wie Bernd Lahno in Bezug auf die Beschreibung des Vertrauens durch die Theorie der rationalen Wahl überzeugend argumentiert, ist die persönliche Einstellung des Vertrauenden gegenüber dem jeweils anderen, die nicht bloß rational ist, aber weitgehend die mit dem Vertrauen verbundenen Erwartungen mitbestimmt (vgl. Lahno 2002, Kap. 5: „Ist Vertrauen eine rationale Erwartung?").

5 Dass allerdings nach dem Zusammenbruch der Sowjetunion, der Auflösung des Warschauer Paktes und der Wiedervereinigung Deutschlands die westliche Sicherheitspolitik nicht, wie von Gorbatschow ausdrücklich gewünscht, in Richtung einer verstärkten KSZE als Institution einer kooperativen Sicherheit in Europa entwickelt wurde, sondern weiterhin die NATO als ein Verteidigungsbündnis und - trotz manchen Veränderungen im Sinne der Kooperation - strukturell eine Institution des Kalten Krieges bestimmend blieb, zeigt allerdings auch, welche Gefahren mit der relativen Einseitigkeit von vertrauensbildenden Maßnahmen verbunden sind. 
Kydd berücksichtigt auch nicht explizit das tragende Klima des Vertrauens, das dessen Schenken maßgeblich beeinflusst. Und er berücksichtigt nicht die Institutionen, die bei der Vertrauensbildung eine wichtige Rolle spielen können: Die KSZE etwa oder die deutsche Ostpolitik seit Willy Brandt werden bei ihm mit keinem Wort erwähnt.

Aber solche wünschenswerte Ergänzungen oder Korrekturen seiner Analyse ändern nichts an seiner Grundthese, dass gerade in Situationen des (Kalten) Krieges und des tiefen Misstrauens das Vertrauen des anderen nicht vorausgesetzt werden kann, sondern mit wiederholten kostspieligen Zeichen gewonnen werden muss, und dass diese kostspieligen Zeichen, genauso wie das Vertrauen, das sie ermöglichen, Wagnisse sind. Denn sie bestehen in einer gewagten Vorleistung, die zuerst unabhängig von der Gegenleistung des anderen erfolgt, obwohl sie sich ihrer Wirkung nie sicher sein kann.

Dieses doppelseitige Wagnis des Vertrauens, das einerseits ohne Garantie des Erfolgs - wenn auch immer mit bestimmten Absicherungen - geschenkt wird, und um das andererseits mit gewagten Vorleistungen geworben wird, scheinen die Bedingungen dafür zu sein, dass eine Sicherheitspolitik durch Kooperation in Zeiten des Konfliktes entstehen, aber auch in Zeiten des Friedens bestehen und sich etablieren kann.

\section{$6 \quad$ Vertrauen und Sicherheit?}

Allerdings ist das Vertrauen allein und von sich aus noch keine Garantie einer sich für alle Beteiligten positiv auswirkenden Sicherheitspolitik. Bereits Georg Simmel (1992, S. 424) erwähnte etwa, dass Vertrauen auch ein besonderes Kennzeichen von Geheimgesellschaften sei, unter denen er ausdrücklich Verbrechergesellschaften 
zählte. Verschwörer und Betrüger müssen sich nämlich vertrauen können, um ihr Geschäft mit einer gewissen Sicherheit durchführen zu können. Aber diese innere Sicherheit einer Zweckgemeinschaft ist für alle anderen eher ein Grund der Unsicherheit.

Die hier vertretene Position besteht also nicht darin zu behaupten, dass Vertrauen als solches zu einer auf Kooperation setzenden Sicherheitspolitik führt. Sie behauptet allerdings, dass eine solche Sicherheitspolitik erstens ohne Vertrauen nicht möglich ist, dass sie aber zweitens die Bedingungen dieses Vertrauens - als eine für sie trotz allem Wagnis annehmbare Basis - nicht einfach vorfindet, sondern weitgehend bilden muss. Im Sinne der Kooperation gehen also sicherheits- und vertrauensbildende Maßnahmen Hand in Hand. Zusammenfassend möchte ich vier dieser Grundbedingungen des Vertrauens besonders betonen:

- Die erste besteht in der Abwägung zwischen einem möglichen Vertrauen und einem nötigen (gesunden) Misstrauen in jeder gegebenen Situation, so dass Vertrauen sich nicht selber gefährdet.

- Die zweite ist die Stärkung des Vertrauens einerseits durch wiederholte Akte des Schenkens unter Bedingungen, unter denen sie nicht zum Scheitern führen, andererseits durch glaubhafte Zeichen der eigenen Vertrauenswürdigkeit, das heißt sowohl durch die Erfüllung der Aufgaben, die einem anvertraut werden, als auch durch kostspielige Signale einer zuverlässigen Kooperationsbereitschaft.

- Die dritte besteht wiederum in der Stärkung der Verbindlichkeit des Vertrauens durch die Einbeziehung von Dritten und die mit ihr einhergehende Erweiterung der Vertrauensbeziehungen.

- Und die vierte liegt in der Stärkung einer sozialen Disposition (Klima, Kultur, Ethos) zum Vertrauen, die sich auf die Möglichkeit einzelner Vertrauensbeziehungen auswirkt. 
Ein Prozess der Vertrauensbildung, der in der Erfüllung dieser Bedingungen besteht, ist meistens ein langwieriger und, besonders im Rahmen von gewaltförmigen Konflikten, ein schwieriger Prozess, der viel Geduld und guten Willen erfordert. Er kann brutal, durch einen einzigen schwerwiegenden Vertrauensbruch (durch den Verrat eines Geheimnisses, den Bruch eines Versprechens oder einen Betrug) zerstört werden. Danach ist der Wiederaufbau des Vertrauens noch schwieriger und brüchiger als vorher. Er kann aber zu einer Form des Zusammenlebens führen, die zwar nicht konfliktfrei ist, in der aber die Konflikte miteinander und auf der Basis des gewonnenen und gepflegten Vertrauens gelöst werden können. Deswegen ist das Vertrauen ein kostbares Gut, mit dem sehr vorsichtig und behutsam umgegangen werden sollte. Deswegen auch ist eine Sicherheitspolitik im Sinne der Kooperation eine Politik des langen Atems und der vielen wiederholten Schritte. Sie ist eine Politik, die nie abgeschlossen ist, wie eine Aufgabe des Sisyphos. Dies mag der Preis dafür sein, dass sie mit mehr Freiheit und weniger Angst zu leben ermöglicht als eine Sicherheitspolitik, die gefahrenzentriert ist.

\section{Literatur}

Baier, Annette. 1994. Moral Prejudices. Essays on Ethics. Cambridge: Harvard University Press.

Bedorf, Thomas. 2003. Dimensionen des Dritten. Sozialphilosophische Modelle zwischen Ethischem und Politischem. München: Fink.

Bollardière, Jacques Pâris de. 1972. Bataille d'Alger, bataille de l'homme, Bar-le-Duc: Desclée de Brouwer.

Czempiel, Ernst-Otto. 2012. KSZE: Die Schlussakte von Helsinki und ihre Auswirkungen. http://universal_lexikon.deacademic.com/263049/ 
KSZE\%3A_Die_Schlussakte_von_Helsinki_und_ihre_Auswirkungen. Zugegriffen: 5. August 2018.

Delhom, Pascal. 2014. Transparenz, Vertrauenswürdigkeit und die Europäische Vertrauenskrise. In Vertrauen und Transparenz - Für ein neues Europa, hrsg. von Alfred Hirsch, Petar Bojanic und Zeljko Radinkovic, 110-138. Belgrad: Institute for Philosophy and Social Theory, University of Belgrade.

Delhom, Pascal. 2015. Frieden mit Verantwortung und Vertrauen. Versuch über den Frieden als soziale Tugend. In Friedensgesellschaften - Zwischen Verantwortung und Vertrauen, hrsg. von Alfred Hirsch und Pascal Delhom, 227-262. Freiburg: Alber.

Delhom, Pascal. 2018. Die Rolle des Gewissens im politisch-ethischen Leitbild des gerechten Friedens. In Gerechter Frieden als politisch-ethisches Leitbild, hrsg. von Sarah Jäger und Jean-Daniel Strub, 107-128. Wiesbaden: Springer VS.

Fukuyama, Francis. 1995. Trust. The Social Virtues and the Creation of Prosperity. New York: Free Press Paperbacks, Simon \& Schuster Inc. Kant, Immanuel. 1968 [1795]. Zum ewigen Frieden. Ein philosophischer Entwurf. In Werkausgabe Band XI: Schriften zur Anthropologie, Geschichtsphilosophie, Politik und Pädagogik 1, hrsg. von Wilhelm Weischedel, 193-251. Frankfurt a. M.: Suhrkamp.

Kydd, Andrew H. 2007. Trust and Mistrust in International Relations. Princeton: Princeton University Press.

Lahno, Bernd. 2002. Der Begriff des Vertrauens. Paderborn: mentis.

Luhmann, Niklas. 2000. Vertrauen. Ein Mechanismus der Reduktion sozialer Komplexität. 4. Aufl. Stuttgart: Lucius \& Lucius.

Montesquieu, Charles de Secondat, Baron de. 1951 [1748]. Vom Geist der Gesetze. Bd. 1. Tübingen: Laupp.

Röttgers, Kurt. 2004. Ethos und Routine. In Kommunikationsmanagement, hrsg. von Günter Bentele, Manfred Piwinger und Gregor Schönborn. Lose Blattsammlung. Neuwied: Luchterhand.

Senghaas, Dieter. 1992. Friedensprojekt Europa. Frankfurt a. M.: Suhrkamp. Simmel, Georg. 1992. Soziologie. Untersuchungen über die Formen der Vergesellschaftung. Gesamtausgabe, Bd. 11. Frankfurt a. M.: Suhrkamp. Sztompka, Piotr. 1999. Trust. A Sociological Theory. Cambridge: Cambridge University Press. 